\section{A) Check for updates}

Cite this: Food Funct., 2019, 10, 3018

\title{
Correction: Free fatty acid release from vegetable and bovine milk fat-based infant formulas and human milk during two-phase in vitro digestion
}

\author{
Jeske H. J. Hageman, (D *a,b Jaap Keijer, (iD a Trine Kastrup Dalsgaard, \\ Lara W. Zeper, (D) ${ }^{a}$ Frédéric Carrière, (D) ${ }^{d}$ Anouk L. Feitsma (D) ${ }^{b}$ and \\ Arie G. Nieuwenhuizen (iD) ${ }^{a}$
}

DOI: $10.1039 /$ c9fo90021g

Correction for 'Free fatty acid release from vegetable and bovine milk fat-based infant formulas and human milk during two-phase in vitro digestion' by Jeske H. J. Hageman et al., Food Funct., 2019, 10,

rsc.li/food-function $2102-2113$

The authors regret that the lipid composition of IF1 was reported incorrectly. The percentage of C18:1n-9 should be $42.3 \%$. Since the incorrect value was also used for some calculations, this also affects some of the results: it increases the total amount of fatty acids in the sample, and consequently the percentage of released FFA is lower. The FFA release (as a percentage of initial composition), both of total FFA and C18:1, is similar for both IFs. One small difference between IF1 and IF2 that was seen when using the incorrect value, i.e. a faster early duodenal digestion for IF1, was found to be no longer statistically significant. This has no consequences for the conclusions of the manuscript.

Page 2106 should read "The human milk samples showed less release of FFA during the gastric phase compared to IF1 and IF2 $(2.0 \pm 0.2 \%$ vs. $4.3 \pm 0.2 \%$ and $4.7 \pm 0.1 \%$ respectively, $p<0.01)$. Compared to the amount of FFA released after the digestion, during the gastric phase $4 \%$ of FFA were released from human milk, about $10 \%$ from IF1, and about 11\% from IF2. Except for 45 minutes $(p=0.04)$, i.e. 15 minutes after the start of the duodenal phase, no differences were found in FFA release between the IFs compared to the human milk samples during this phase. The total release of FAs at the end of the digestion, as percentage of initial composition, was found to be similar for the different samples $(43.9 \pm 2.0 \%, 42.2 \pm 1.4 \%$, and $52.3 \pm 4.5 \%$ for IF1, IF2 and human milk respectively, $p=0.14)$.

The correspondingly updated Fig. 2B, Fig. 5K, Table 2 and Table 3 are as presented below.

\footnotetext{
${ }^{a}$ Human and Animal Physiology, Wageningen University, de Elst 1, 6708 WD Wageningen, the Netherlands. E-mail: jeske.hageman@frieslandcampina.com

${ }^{b}$ FrieslandCampina, Stationsplein 1, 3818 LE Amersfoort, the Netherlands

${ }^{c}$ Department of Food Science, Aarhus University, Blichers Allé 20, DK-8830 Tjele Aarhus, Denmark

${ }^{d}$ Aix Marseille Univ, CNRS, Bioénergetique et Ingénierie des Protéines UMR7281, 31 chemin Joseph Aiguier, 13402 Marseille, France
} 
B

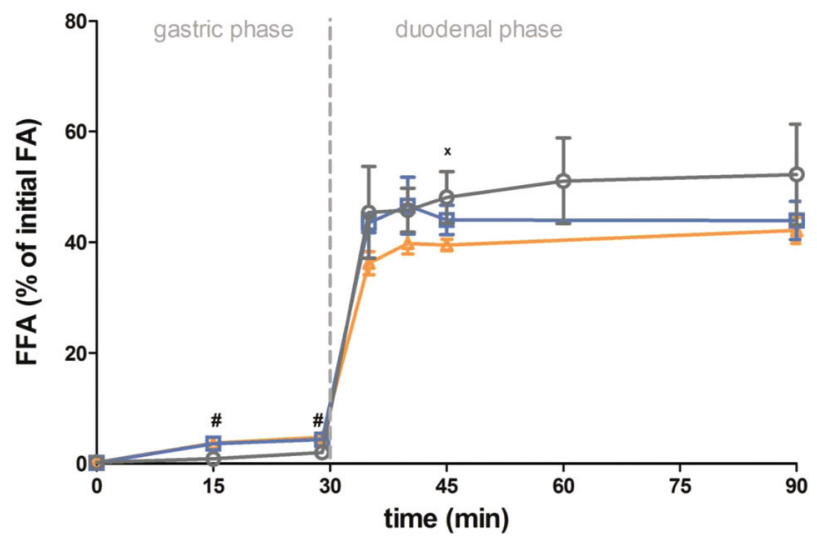

Fig. 2 B: The lipolysis of infant formulas and human milk (mean \pm SD). B: Percentage of free fatty acids (FFA) in percentage of total esterified fatty acids (FA) in initial TAGs, during in vitro digestion (mean \pm SD). At time point 0 the FFA percentages were $0.15 \%, 0.20 \%$, and $0.22 \%$ for IF- 1 , IF- 2 and human milk respectively. The vertical striped line indicates the start of the duodenal phase following the gastric phase. The grey line $(O)$ represents the average of the human milk samples, the blue line $(\square)$ represents IF1, and the orange line $(\Delta)$ represents IF2. \# Significant difference between both IFs and human milk, $x$ significant difference between IF2 and human milk $(p<0.05)$, separate time points were compared with one-way ANOVA, followed by Bonferroni post hoc test.

\section{K}

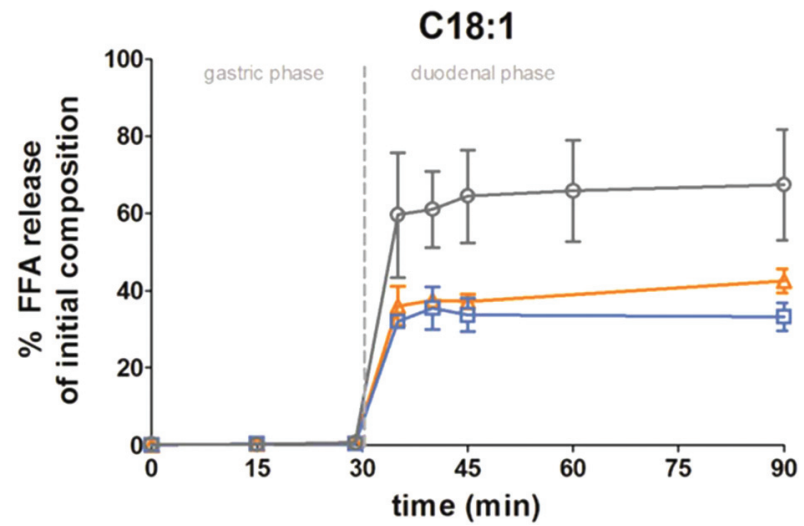

Fig. 5 K: Release of individual fatty acids during in vitro digestion, expressed as percentage of their initial FA content present in IFs and human milk (mean \pm SD) (K) C18:1. The vertical striped line indicates the start of the duodenal phase following the gastric phase. The grey line $(O)$ represents human milk, the blue line $(\square)$ represents IF1, and the orange line $(\Delta)$ represents IF2. 
Table 2 Initial fatty acid composition of the infant formula products and human milk samples (in \% of total FAMEs)

\begin{tabular}{|c|c|c|c|c|c|c|}
\hline Fatty acid & IF1 & IF2 & Human milk-1 & Human milk-2 & Human milk-3 & Human milk-4 \\
\hline $\mathrm{C} 4: 0$ & $<0.1$ & 2.6 & $<0.1$ & ND & $<0.1$ & $<0.1$ \\
\hline C6:0 & $<0.1$ & 1.6 & $<0.1$ & ND & $<0.1$ & $<0.1$ \\
\hline C8:0 & 0.6 & 1.2 & $<0.1$ & $<0.1$ & 0.2 & 0.3 \\
\hline C10:0 & 0.5 & 2.1 & 0.9 & 0.7 & 1.6 & 1.6 \\
\hline C11:0 & $<0.1$ & 0.3 & $<0.1$ & $<0.1$ & $<0.1$ & $<0.1$ \\
\hline C12:0 & 7.0 & 4.3 & 3.8 & 3.1 & 7.8 & 5.6 \\
\hline C14:0 & 3.0 & 8.2 & 6.1 & 6.7 & 10.0 & 5.9 \\
\hline C15:0 & $<0.1$ & 0.7 & 0.5 & 0.6 & 0.4 & 0.3 \\
\hline C16:0 & 24.7 & 22.8 & 30.0 & 28.3 & 21.0 & 18.4 \\
\hline C18:0 & 3.1 & 7.1 & 8.1 & 9.4 & 6.6 & 4.7 \\
\hline C18:1 n-9cis & 42.3 & 25.9 & 30.3 & 29.8 & 32.2 & 30.8 \\
\hline C18:2 n-6 & 12.9 & 12.0 & 10.5 & 10.0 & 10.3 & 20.6 \\
\hline C18:3 n-3 & 1.8 & 1.4 & 0.8 & 0.8 & 0.8 & 1.6 \\
\hline Total SC/MCFA & 1.4 & 7.8 & 1.3 & 0.9 & 2.1 & 2.2 \\
\hline Total LCFA & 94.9 & 82.4 & 90.1 & 88.7 & 89.1 & 87.9 \\
\hline Total SFA & 39.3 & 50.9 & 49.8 & 49.0 & 47.9 & 37.1 \\
\hline Total MUFA & 42.3 & 25.9 & 30.3 & 29.8 & 32.2 & 30.8 \\
\hline Total PUFA & 14.7 & 13.4 & 11.3 & 10.8 & 11.1 & 22.2 \\
\hline
\end{tabular}

Table 3 Release of the individual fatty acids, as percentage of their initial FA content present in IFs and human milk (mean \pm SEM) upon digestion of IF1, IF2 and human milk. P-Values represent the results of one-way ANOVA, or in case of C4:0, C11:0 and C15:0 the results of a Student's t-test, difference between products is indicated with letters (Bonferroni post hoc test). nd, not detected

\begin{tabular}{|c|c|c|c|c|c|c|c|c|c|c|c|c|}
\hline \multirow[b]{2}{*}{ Fatty acid (\%) } & \multicolumn{4}{|c|}{ Gastric phase } & \multicolumn{4}{|c|}{ Duodenal phase } & \multicolumn{4}{|c|}{ Total digestion } \\
\hline & IF1 & IF2 & Human milk & $p$-Value & IF1 & IF-2 & Human milk & $p$-Value & IF1 & IF2 & Human milk & $p$-Value \\
\hline $\mathrm{C} 4: 0$ & nd & $13.7 \pm 1.8^{\mathrm{a}}$ & $0.0 \pm 0.0^{\mathrm{b}}$ & $<0.01$ & nd & $41.1 \pm 7.6$ & $22.7 \pm 3.7$ & 0.10 & nd & $54.7 \pm 5.8^{\mathrm{a}}$ & $22.7 \pm 3.7^{b}$ & 0.01 \\
\hline C6:0 & $33.5 \pm 0.7^{\mathrm{b}}$ & $24.9 \pm 2.4^{\mathrm{a}}$ & $20.1 \pm 3.7^{\mathrm{a}}$ & 0.07 & $3.9 \pm 0.9^{\mathrm{a}}$ & $20.7 \pm 1.5^{b}$ & $40.9 \pm 3.1^{\mathrm{c}}$ & $<0.01$ & $37.4 \pm 1.1^{\mathrm{a}}$ & $46.6 \pm 3.6^{\mathrm{a}, \mathrm{b}}$ & $60.5 \pm 1.8^{b}$ & 0.02 \\
\hline C8:0 & $55.5 \pm 0.8$ & $34.1 \pm 0.4$ & $52.4 \pm 14.8$ & 0.38 & $1.8 \pm 0.8^{\mathrm{a}}$ & $11.3 \pm 0.7^{\mathrm{a}}$ & $53.8 \pm 15.8^{\mathrm{b}}$ & $<0.01$ & $57.3 \pm 1.1$ & $45.4 \pm 0.0$ & $106.2 \pm 22.0$ & 0.06 \\
\hline C10:0 & $46.8 \pm 0.6$ & $26.5 \pm 1.0$ & $33.4 \pm 8.2$ & 0.13 & $14.1 \pm 4.5^{\mathrm{a}}$ & $13.5 \pm 2.7^{\mathrm{a}}$ & $51.6 \pm 19.6^{\mathrm{b}}$ & $<0.01$ & $60.9 \pm 3.0$ & $40.0 \pm 0.9$ & $85.0 \pm 18.0$ & 0.11 \\
\hline C11:0 & nd & $3.0 \pm 0.1^{\mathrm{a}}$ & $8.5 \pm 1.4^{b}$ & 0.02 & nd & $3.6 \pm 0.3^{\mathrm{a}}$ & $10.4 \pm 2.1^{\mathrm{b}}$ & $<0.01$ & nd & $6.6 \pm 0.2^{\mathrm{a}}$ & $19.0 \pm 1.8^{\mathrm{b}}$ & $<0.01$ \\
\hline C12:0 & $24.9 \pm 1.2^{\mathrm{a}}$ & $10.9 \pm 0.2^{\mathrm{b}}$ & $11.6 \pm 2.2^{\mathrm{b}}$ & $<0.01$ & $26.0 \pm 3.9^{\mathrm{a}}$ & $25.1 \pm 1.5^{\mathrm{a}}$ & $55.5 \pm 12.9^{\mathrm{b}}$ & $<0.01$ & $50.9 \pm 2.3$ & $36.1 \pm 0.9$ & $67.1 \pm 8.7$ & 0.33 \\
\hline C14:0 & $3.1 \pm 0.3^{\mathrm{a}}$ & $1.7 \pm 0.0^{\mathrm{b}}$ & $0.4 \pm 0.1^{\mathrm{c}}$ & $<0.01$ & $40.2 \pm 2.0^{\mathrm{a}}$ & $26.9 \pm 1.4^{b}$ & $33.5 \pm 5.7^{\mathrm{a}, \mathrm{b}}$ & 0.01 & $43.3 \pm 1.3^{\mathrm{a}}$ & $28.6 \pm 0.8^{b}$ & $33.9 \pm 2.9^{\mathrm{a}, \mathrm{b}}$ & $<0.01$ \\
\hline C15:0 & nd & $2.1 \pm 0.2$ & $3.3 \pm 0.9$ & 0.35 & nd & $23.1 \pm 1.0$ & $18.7 \pm 4.5$ & 0.16 & nd & $25.2 \pm 0.6$ & $24.6 \pm 2.6$ & 0.83 \\
\hline C16:0 & $1.8 \pm 0.1^{\mathrm{a}}$ & $1.5 \pm 0.1^{\mathrm{a}}$ & $0.2 \pm 0.1^{b}$ & $<0.01$ & $55.4 \pm 6.7^{\mathrm{a}}$ & $44.5 \pm 5.7^{\mathrm{a}, \mathrm{b}}$ & $31.7 \pm 6.0^{b}$ & $<0.01$ & $57.2 \pm 4.0^{\mathrm{a}}$ & $46.0 \pm 3.2^{\mathrm{a}, \mathrm{b}}$ & $31.8 \pm 3.0^{\mathrm{b}}$ & $<0.01$ \\
\hline C18:0 & $0.5 \pm 0.3$ & $0.7 \pm 0.4$ & $0.2 \pm 0.2$ & 0.51 & $76.9 \pm 7.7$ & $54.1 \pm 2.7$ & $63.5 \pm 13.6$ & 0.07 & $77.4 \pm 4.2$ & $54.8 \pm 1.6$ & $63.7 \pm 6.9$ & 0.07 \\
\hline C18:1 & $0.5 \pm 0.1$ & $0.6 \pm 0.1$ & $0.7 \pm 0.1$ & 0.41 & $32.7 \pm 3.5^{\mathrm{a}}$ & $41.9 \pm 3.2^{\mathrm{a}}$ & $66.6 \pm 14.0^{\mathrm{b}}$ & $<0.01$ & $33.2 \pm 2.1^{\mathrm{a}}$ & $42.5 \pm 1.8^{\mathrm{a}}$ & $67.4 \pm 7.1^{b}$ & $<0.01$ \\
\hline C18:2 & $0.4 \pm 0.1^{\mathrm{a}}$ & $0.4 \pm 0.1^{\mathrm{a}}$ & $1.0 \pm 0.2^{\mathrm{b}}$ & 0.02 & $33.0 \pm 4.6^{\mathrm{a}}$ & $32.6 \pm 3.0^{\mathrm{a}}$ & $61.1 \pm 12.4^{\mathrm{b}}$ & $<0.01$ & $33.4 \pm 2.6^{\mathrm{a}}$ & $32.9 \pm 1.7^{\mathrm{a}}$ & $62.3 \pm 6.3^{b}$ & $<0.01$ \\
\hline C18:3 & $1.0 \pm 0.2^{\mathrm{a}}$ & $1.2 \pm 0.2^{\mathrm{a}}$ & $2.9 \pm 0.5^{b}$ & 0.02 & $35.2 \pm 4.1^{\mathrm{a}}$ & $36.1 \pm 3.1^{\mathrm{a}}$ & $68.2 \pm 12.6^{\mathrm{b}}$ & $<0.01$ & $36.2 \pm 2.5^{\mathrm{a}}$ & $37.4 \pm 1.9^{\mathrm{a}}$ & $71.1 \pm 6.7^{\mathrm{b}}$ & $<0.01$ \\
\hline
\end{tabular}

The Royal Society of Chemistry apologises for these errors and any consequent inconvenience to authors and readers. 